\title{
Radiative lepton flavor violating decays in the Randall Sundrum background with localized leptons
}

\author{
E. O. Iltan * \\ Physics Department, Middle East Technical University \\ Ankara, Turkey
}

\begin{abstract}
We study the radiative lepton flavor violating $l_{i} \rightarrow l_{j} \gamma$ decays in the two Higgs doublet model, respecting the Randall Sundrum scenario and estimate the contributions of the KK modes of left (right) handed charged lepton doublets (singlets) on the branching ratios. We observe that the branching ratios are sensitive to the contributions of the charged lepton KK modes.
\end{abstract}

${ }^{*}$ E-mail address: eiltan@newton.physics.metu.edu.tr 


\section{Introduction}

The flavor violation (FV) is among the most interesting physical phenomena to search the standard model (SM) and its beyond. The lepton FV is worthwhile to study since the leptonic decays are clean and rich from the theoretical point of view. They are clean because of the fact that they are free from strong interactions. On the other hand, they are rich since they exist at least in the loop level. The lepton $\mathrm{FV}$ is tiny in the extended SM, with non zero neutrino masses and the theoretical branching ratios (BRs) of lepton flavor violating (LFV) decays are too small to reach the experimental limits. Therefore, one needs to search more fundamental models to pull the theoretical values to the current experimental results. The radiative $l_{i} \rightarrow l_{j} \gamma$, $i \neq j$ decays are among the LFV interactions and the current experimental upper limits of the BRs of the decays $\mu \rightarrow e \gamma, \tau \rightarrow e \gamma$ and $\tau \rightarrow \mu \gamma$, read $1.2 \times 10^{-11}$ [1], $3.9 \times 10^{-7}$ [2] and $1.1 \times 10^{-6}\left(9.0 \times 10^{-8} ; 6.8 \times 10^{-8}, 90 \% C L\right)$ [3] ([4]; [5]), respectively. Furthermore, a new experiment at PSI has been described [6] in order to search the $\mu \rightarrow e \gamma$ decay and the aim is to reach to a sensitivity of $\mathrm{BR} \sim 10^{-14}$. At present, this experiment (PSI-R-99-05 Experiment) is still running in the MEG [7].

The general two Higgs doublet model (2HDM) with the flavor changing neutral currents (FCNCs) at tree level is the most primitive model beyond the SM to enhance the BRs of the LFV decays under consideration and the lepton FV is induced by the internal new neutral Higgs bosons, $h^{0}$ and $A^{0}$. The Yukawa couplings appear as free parameters and they can be determined by the experimental data. These decays were examined in the framework of the 2HDM in [8, 9, 10, 11, 12, 13, 14]. Besides the theoretical calculations based on the 2HDM, they were studied in the supersymmetric models [15]-[21], in a model independent way [22] and, recently, they were analyzed in the framework of 2HDM and the supersymmetric model [23].

The present work is devoted to the analysis of the radiative $l_{i} \rightarrow l_{j} \gamma, i \neq j$ decays in the 2HDM1], including a single extra dimension, respecting the Randall Sundrum scenario [24, 25]. The extra dimensions are introduced to solve the hierarchy problem between weak and Planck scales. The Randall Sundrum model (the RS1 model) is based on the non-factorizable geometry of the extra dimension that the gravity is localized on a $4 \mathrm{D}$ brane boundary, so called hidden (Planck) brane, and the other fields, including the SM fields, live on another 4D brane boundary, so called the visible $(\mathrm{TeV})$ brane. The difference of induced metrics on these boundaries are carried by a warp factor that is an exponential function of the compactified radius in the extra

\footnotetext{
${ }^{1}$ Here, we assume that the Cabibbo-Kobayashi-Maskawa (CKM) type matrix in the leptonic sector does not exist, the charged flavor changing (FC) interactions vanish and the lepton FV comes from the internal new neutral Higgs bosons, $h^{0}$ and $A^{0}$.
} 
dimension and it connects two effective scales, the Planck scale $M_{P l}$ and the weak scale $m_{W}$. In the case that some of the SM fields are accessible to the extra dimension, the phenomenology becomes richer and there are various work done respecting such scenarios in the literature [26][44]. In [28, 29] the behavior of $U(1)$ gauge boson, living in the extra dimension of the RS1 background, has been studied. In these works, it is observed that the massless mode of the gauge field is not localized in the extra dimension and its KK excitations have large couplings to boundaries. Since it is necessary to push the visible scale to energies greater than TeV for a perturbative theory, this is not a phenomenologically favorable scenario. The brane localized mass terms for scalar fields have been considered to get small couplings of KK modes with the boundaries [30]. In this case, these mass terms change the boundary conditions and the zero mode localized solution is obtained. [31] is devoted to the localized $U(1)_{Y}$ gauge field with bulk and boundary mass terms. In [32], the KK excitations of $\mathrm{W}$ and $\mathrm{Z}$ bosons at the LHC are studied. A further approach is to consider that the fermions are also accessible to the extra dimension and to explain the fermion mass hierarchy with the addition of Dirac mass term to the Lagrangian. The bulk fermions are considered in the RS1 background and the fermion mass hierarchy, coming from the possible fermion field locations, is studied in [30, 33, 34, 35]. In [36] this hierarchy is analyzed, by taking that the Higgs field has an exponential profile around the $\mathrm{TeV}$ brane. [37] is devoted to an extensive work on the bulk fields in various multi-brane models. The quark and lepton FV, which is based on the different locations of the fermion fields in the extra dimension, is extensively studied in [40, 41] and in these works, it is considered that the FV is carried by the Yukawa interactions, coming from the SM Higgs-fermion-fermion vertices. In [42], the high precision measurements of top pair production at the ILC is addressed by considering that the fermions are localized in the bulk of RS1 background. In recent works [43, 44, the various experimental FCNC constraints and the electro weak precision tests for the location parameters of the fermions in the extra dimension are discussed.

In this work, we study the $\mathrm{LFV} l_{i} \rightarrow l_{j} \gamma$ decays in the RS1 background with the assumption that the leptons and gauge fields are accessible to the extra dimension. Furthermore, we consider that the lepton fields are localized in the extra dimension and their mass hierarchy is based on the lepton field location in the extra dimension.

The paper is organized as follows: In Section 2, we present the BRs of LFV interactions $l_{i} \rightarrow l_{j} \gamma$ in the $2 \mathrm{HDM}$, by considering that the lepton fields are localized in the extra dimension of RS1 background. Section 3 is devoted to discussion and our conclusions. 


\section{LFV $l_{i} \rightarrow l_{j} \gamma$ decays in the Randall Sundrum back- ground with localized lepton fields}

The radiative LFV decays exist in the loop level and, therefore, the theoretical expressions of their physical quantities contain number of free parameters of the model used. The theoretical work done on these decays ensures considerable information on the parameters of the model, with the help of the experimental measurements. In the framework of the SM, the numerical values of the BRs of these decays are far from experimental limits. This forces one to consider new models beyond the SM and the 2HDM, with the FCNCs at tree level, is one of the candidate. The new neutral Higgs bosons in the 2HDM switch on the LFV interactions and the new Yukawa couplings, arising from lepton-lepton-scalar interactions, play the main role in the calculation of the physical quantities related to these decays.

Addition of the contributions coming from the extra dimension(s) further enhances the BRs of the decays studied and, in the present work, we study these effects in the RS1 background, by assuming that the charged leptons and the gauge bosons are accessible to the extra dimension. In the RS1 model, the extra dimension is curved and the metric reads

$$
d s^{2}=e^{-2 \sigma} \eta_{\mu \nu} d x^{\mu} d x^{\nu}-d y^{2}
$$

where $\sigma=k|y|$ and $k$ is the bulk curvature constant. Here the exponential $e^{-k|y|}$, with $y=R|\theta|$, is the warp factor 2 that is responsible for the hierarchy and $R$ is the compactification radius in the extra dimension that is compactified onto $S^{1} / Z_{2}$ orbifold, having two boundaries, the hidden (Planck) brane and the visible (TeV) brane. In the RS1 background, the gravity is considered to be localized on the hidden brane and to be extended into the bulk with varying strength, on the other hand, the SM fields live in the visible brane. By choosing opposite and equal tensions on the hidden and visible branes, the low energy effective theory has flat 4D spacetime, even if the $5 \mathrm{D}$ cosmological constant is non vanishing. In the present work, we assume that the gauge fields and the fermions are also accessible to the extra dimension. The addition of Dirac mass term to the lagrangian of bulk fermions results in the fermion localization in the extra dimension [27, 29, 30, 32, 33, 35, 36. Since the fermions have two possible transformation properties under the orbifold $Z_{2}$ symmetry, $Z_{2} \psi= \pm \gamma_{5} \psi$, the combination $\bar{\psi} \psi$ is odd and, in order to construct the $Z_{2}$ invariant mass term, one needs $Z_{2}$ odd scalar field to be coupled. Therefore, the mass term reads

$$
\mathcal{S}_{m}=-\int d^{4} x \int d y \sqrt{g} m(y) \bar{\psi} \psi,
$$

\footnotetext{
${ }^{2}$ The warp factor causes that all mass terms are rescaled on the visible brane for $\theta=\pi$.
} 
where $m(y)=m \frac{\sigma^{\prime}(y)}{k}$ with $\sigma^{\prime}(y)=\frac{d \sigma}{d y}$ and $g=\operatorname{Det}\left[g_{M N}\right]=e^{-8 \sigma}, M, N=0,1, \ldots, 4$. By expanding the bulk fermion as

$$
\psi\left(x^{\mu}, y\right)=\frac{1}{\sqrt{2 \pi R}} \sum_{n=0}^{\infty} \psi^{(n)}\left(x^{\mu}\right) e^{2 \sigma} \chi_{n}(y),
$$

and using the normalization

$$
\frac{1}{2 \pi R} \int_{-\pi R}^{\pi R} d y e^{\sigma} \chi_{n}(y) \chi_{m}(y)=\delta_{n m}
$$

the Dirac equation is solved for the zero mode fermion and it is obtained as follows:

$$
\chi_{0}(y)=N_{0} e^{-r \sigma}
$$

where $r=m / k$ and the normalization constant $N_{0}$ reads

$$
N_{0}=\sqrt{\frac{k \pi R(1-2 r)}{e^{k \pi R(1-2 r)}-1}} .
$$

The appropriately normalized solution

$$
\chi_{0}^{\prime}(y)=e^{-\frac{\sigma}{2}} \chi_{0}(y)
$$

is localized in the extra dimension and the localization is regulated by the parameter $r$. This solution is localized near the hidden (visible) brane if $r>\frac{1}{2}\left(r<\frac{1}{2}\right)$ and it has a constant profile if $r=\frac{1}{2}$.

To construct the SM fermions, one needs to consider $S U(2)_{L}$ doublet $\psi_{L}$ and singlet $\psi_{R}$ with separate $Z_{2}$ projection conditions: $Z_{2} \psi_{R}=\gamma_{5} \psi_{R}$ and $Z_{2} \psi_{L}=-\gamma_{5} \psi_{L}$ (see for example [27]). This is the case that the zero mode fermions can get mass through the $Z_{2}$ invariant left handed fermion-right handed fermion-Higgs interaction, $\bar{\psi}_{R} \psi_{L} H 3$ and the location parameters for fermion fields, different flavors and their left and right handed parts, can be chosen so that this interaction creates the current masses of fermions. With the assumption that the Higgs field lives on the visible brane, the masses of fermions are calculated by using the integral

$$
m_{i}=\frac{1}{2 \pi R} \int_{-\pi R}^{\pi R} d y \lambda_{5} \chi_{i L 0}(y) \chi_{i R 0}(y)<H>\delta(y-\pi R)
$$

where $\lambda_{5}$ is the coupling in five dimensions and it can be parametrized in terms of the one in four dimensions, the dimensionless coupling $\lambda, \lambda_{5}=\lambda / \sqrt{k}$. Here the expectation value of the Higgs field $\langle H\rangle$ reads $\langle H\rangle=v / \sqrt{k}$ where $v$ is the vacuum expectation value 4 . Now, we choose three different sets of location of charged lepton fields in order to obtain the masses of different flavors.

\footnotetext{
${ }^{3}$ Here, we consider different location parameters $r$ for each left handed and right handed part of different flavors.

${ }^{4}$ We take $v=0.043 M_{P l}$ to provide the measured gauge boson masses [35] and choose $k R=10.83$ in order to get the correct effective scale on the visible brane, i.e., $M_{W}=e^{-\pi k R} M_{p l}$ is of the order of TeV.
} 


\begin{tabular}{|c|cc|cc|cc|}
\hline & \multicolumn{2}{|c|}{ SET I } & \multicolumn{2}{c|}{ SET II } & \multicolumn{2}{c|}{ SET III } \\
\hline \hline & $r_{L}$ & $r_{R}$ & $r_{L}$ & $r_{R}$ & $r_{L}$ & $r_{R}$ \\
\hline \hline $\mathrm{e}$ & 0.6710 & 0.6710 & -0.4900 & 0.8800 & -1.0000 & 0.8860 \\
\hline$\mu$ & 0.5826 & 0.5826 & -0.4900 & 0.7160 & -1.0000 & 0.7230 \\
\hline$\tau$ & 0.5273 & 0.5273 & -0.4900 & 0.6249 & -1.0000 & 0.6316 \\
\hline \hline
\end{tabular}

Table 1: Three possible locations of charged lepton fields. Here $r_{L}$ and $r_{R}$ are left handed and right handed lepton field location parameters, respectively.

In Set I, the left and right handed fields of the same flavor have the same location. In set II and III, we choose the left handed charged lepton locations the same for each flavor and we estimate the left handed charged lepton locations by respecting their masses. Notice that, in the third set, we take the left handed charged lepton locations nearer to the visible brane.

Now we would like to present the part of the action responsible for the LFV interactions in 5 dimension:

$$
\mathcal{S}_{Y}=\int d^{5} x \sqrt{-g}\left(\xi_{5 i j}^{E} \bar{l}_{i L} \phi_{2} E_{j R}+\text { h.c. }\right) \delta(y-\pi R)
$$

where $L$ and $R$ denote chiral projections $L(R)=1 / 2\left(1 \mp \gamma_{5}\right), \phi_{2}$ is the new scalar doublet, $l_{i L}$ $\left(E_{j R}\right)$ are lepton doublets (singlets), $\xi_{5 i j}^{E}$, with family indices $i, j$, are the Yukawa couplings in five dimensions, which induce the FV interactions in the leptonic sector. We assume that the Higgs doublet $\phi_{1}$, that lives on the visible brane, has non-zero vacuum expectation value to ensure the ordinary masses of the gauge fields and the fermions, however, the second doublet, that lies also on the visible brane, has no vacuum expectation value:

$$
\phi_{1}=\frac{1}{\sqrt{2}}\left[\left(\begin{array}{c}
0 \\
v+H^{0}
\end{array}\right)+\left(\begin{array}{c}
\sqrt{2} \chi^{+} \\
i \chi^{0}
\end{array}\right)\right] ; \phi_{2}=\frac{1}{\sqrt{2}}\left(\begin{array}{c}
\sqrt{2} H^{+} \\
H_{1}+i H_{2}
\end{array}\right),
$$

and the vacuum expectation values are

$$
<\phi_{1}>=\frac{1}{\sqrt{2}}\left(\begin{array}{l}
0 \\
v
\end{array}\right) ;<\phi_{2}>=0 .
$$

By considering the gauge and $C P$ invariant Higgs potential which spontaneously breaks $S U(2) \times$ $U(1)$ down to $U(1)$ as:

$$
\begin{aligned}
V\left(\phi_{1}, \phi_{2}\right) & =c_{1}\left(\phi_{1}^{+} \phi_{1}-v^{2} / 2\right)^{2}+c_{2}\left(\phi_{2}^{+} \phi_{2}\right)^{2} \\
& +c_{3}\left[\left(\phi_{1}^{+} \phi_{1}-v^{2} / 2\right)+\phi_{2}^{+} \phi_{2}\right]^{2}+c_{4}\left[\left(\phi_{1}^{+} \phi_{1}\right)\left(\phi_{2}^{+} \phi_{2}\right)-\left(\phi_{1}^{+} \phi_{2}\right)\left(\phi_{2}^{+} \phi_{1}\right)\right] \\
& +c_{5}\left[\operatorname{Re}\left(\phi_{1}^{+} \phi_{2}\right)\right]^{2}+c_{6}\left[\operatorname{Im}\left(\phi_{1}^{+} \phi_{2}\right)\right]^{2}+c_{7},
\end{aligned}
$$


with constants $c_{i}, i=1, \ldots, 7, H_{1}$ and $H_{2}$ are obtained as the mass eigenstates $h^{0}$ and $A^{0}$ respectively, since no mixing occurs between two CP-even neutral bosons $H^{0}$ and $h^{0}$ in the tree level. With the choice eq.(11) and the potential eq.(12), the SM particles can be collected in the first doublet and the new particles in the second on 5 . Since the lepton fields are accesible to extra dimension, the lepton doublets $l_{i L}$ and singlets $E_{j R}$ are expanded as

$$
\begin{aligned}
l_{i L}\left(x^{\mu}, y\right) & =\frac{1}{\sqrt{2 \pi R}} e^{2 \sigma} l_{i L}^{(0)}\left(x^{\mu}\right) \chi_{i L 0}(y) \\
& +\frac{1}{\sqrt{2 \pi R}} \sum_{n=1}^{\infty} e^{2 \sigma}\left(l_{i L}^{(n)}\left(x^{\mu}\right) \chi_{i L n}^{l}(y)+l_{i R}^{(n)}\left(x^{\mu}\right) \chi_{i R n}^{l}(y)\right) \\
E_{j R}\left(x^{\mu}, y\right) & =\frac{1}{\sqrt{2 \pi R}} e^{2 \sigma} E_{j R}^{(0)}\left(x^{\mu}\right) \chi_{j R 0}(y) \\
& +\frac{1}{\sqrt{2 \pi R}} \sum_{n=0}^{\infty} e^{2 \sigma}\left(E_{j R}^{(n)}\left(x^{\mu}\right) \chi_{j R n}^{E}(y)+E_{j L}^{(n)}\left(x^{\mu}\right) \chi_{j L n}^{E}(y)\right) .
\end{aligned}
$$

The zero mode leptons $\chi_{i L 0}(y)$ and $\chi_{j R 0}(y)$ are given in eq. (5) with the replacements $r \rightarrow r_{i L}$ and $r \rightarrow r_{i R}$, respectively. For the effective Yukawa coupling $\xi_{i j}^{E}$, we need to integrate out the Yukawa interaction eq.(91) over the fifth dimension by taking the zero mode lepton doublets, singlets, and neutral Higgs fields $S=h^{0}, A^{0}$ :

$$
\begin{aligned}
V_{R L i j}^{0} & =\frac{\xi_{5 i j}^{E}}{2 \pi R} \int_{-\pi R}^{\pi R} d y \chi_{i R 0}(y) \chi_{j L 0}(y) \delta(y-\pi R) \\
& =\frac{e^{-k \pi R\left(r_{i R}+r_{j L}\right)} k \sqrt{\left(1-2 r_{i R}\right)\left(1-2 r_{j L}\right)}}{\sqrt{\left(e^{k \pi R\left(1-2 r_{i R}\right)}-1\right)\left(e^{k \pi R\left(1-2 r_{j L}\right)}-1\right)}} \xi_{5 i j}^{E} .
\end{aligned}
$$

At this stage, we consider two different scenarios. At first, we assume that the hierarchy of new Yukawa couplings comes from the lepton field localization and, by considering that the left and right handed fields of the same flavor have the same location (Set I), we construct the Yukawa coupling $\xi_{i j}^{E}$ and its hermitian conjugate as follows:

$$
\xi_{i j}^{E}\left(\left(\xi_{i j}^{E}\right)^{\dagger}\right)=\frac{V_{R L(L R) i j}^{0}}{V_{R L(L R) \mu \tau}^{0}} \alpha .
$$

Here, we fix the new neutral Higgs $S-\tau-\mu$ coupling (renamed as $\alpha$ ) in four dimensions, and we estimate the other couplings, respecting the possible locations of the charged leptons which are calculated by considering their mass hierarchy 6 . The idea behind is to consider the

\footnotetext{
${ }^{5}$ In the case that the second Higgs doublet has also a vacuum expectation value, there would be a mixing between the CP even neutral Higgs bosons and there appears an additional free parameter, the mixing angle, which should be restricted. In general, this mixing is considered weak and, our choice of Higgs doublets results in vanishing mixing between CP even neutral Higgs bosons. This is the case that one free parameter is dropped without loosing the crucial property of the model, namely, the FV at tree level

${ }^{6}$ This case is similar to Sher anzats [45] that the couplings are defined as $\xi_{i j}^{E} \sim \sqrt{m_{i} m_{j}}$
} 
$\tau-\mu$ coupling in four dimensions as non-zero in the case of $S$-charged lepton-charged lepton interaction (zero for the case of SM Higgs-charged lepton-charged lepton interaction since the off diagonal entries of the SM Higgs-charged lepton-charged lepton couplings in five dimensions vanish, in our choice) and it is fixed by embedding the contribution coming from the mixing due to the different locations of $\mathrm{L}(\mathrm{R})$ handed muon-R(L) handed tau 7 into the number $\alpha$, with the choice of flavor dependent coupling in five dimensions. In other words, the flavor dependence due to the different locations of $\mathrm{L}(\mathrm{R})$ handed muon, $\mathrm{R}(\mathrm{L})$ handed tau is compensated by the flavor dependence of the coupling in five dimensions and, at the end, we get a fixed number $\alpha$. As a second scenario, we embed the vertex factor $V_{R L(L R) i j}^{0}$ into the coupling $\xi_{i j}^{E}\left(\left(\xi_{i j}^{E}\right)^{\dagger}\right)$. In this case, similar to the previous one, the coupling $\xi_{5 i j}^{E}$ in five dimension is flavor dependent and it is regulated in such a way that the overall quantity $V_{R L}^{0}(L R) i j$ is pointed to the chosen numerical value of $\xi_{i j}^{E}\left(\left(\xi_{i j}^{E}\right)^{\dagger}\right)$. In this scenario, the hierarchy of new Yukawa couplings is not related to the lepton field locations. Furthermore, the strengths of $S$-KK mode charged lepton-charged lepton couplings are regulated by the locations of the lepton fields. In our second scenario, we use two different sets, Set II and Set III, for the locations of the lepton fields. Notice that in both scenarios, the FV is carried by the new Yukawa couplings in four dimensions and the effect of extra dimension is the enhancement in the physical quantities of the processes studied 8

Since the radiative LFV decays under consideration exist at least in the one loop level, the $S$-charged lepton-KK charged lepton vertices arise and these vertex factors should be taken into account. The $Z_{2}$ the projection condition $Z_{2} \psi=-\gamma_{5} \psi$, used in constructing the left handed fields on the branes, results in that the left handed zero mode appears, the left (right) handed KK modes appear (disappear) on the branes, with the boundary conditions due to the Dirac mass term in the action eq.(2):

$$
\begin{aligned}
\left(\frac{d}{d y}-m\right) \chi_{i L n}^{l}\left(y_{0}\right) & =0 \\
\chi_{i R n}^{l}\left(y_{0}\right) & =0
\end{aligned}
$$

where $y_{0}=0$ or $\pi R$. Using the Dirac equation for KK mode leptons one gets the left handed lepton $\chi_{i L n}^{l}(y)$ that lives on the visible brane as

$$
\chi_{i L n}^{l}(y)=N_{L n} e^{\sigma / 2}\left(J_{\frac{1}{2}-r}\left(e^{\sigma} x_{n L}\right)+c_{L} Y_{\frac{1}{2}-r}\left(e^{\sigma} x_{n L}\right)\right),
$$

\footnotetext{
${ }^{7}$ Notice that we choose $c_{L}=c_{R}$ for this case.

${ }^{8}$ These scenarios are different than the ones presented in the works [40, 41] where the sources of the FV are explicitly the parameters related to the different locations of the fermion fields in the extra dimension. In these works, it is considered that the FV is carried by the Yukawa interactions, coming from the SM Higgs-fermionfermion vertices with the help of the coupling in five dimensions, including non-zero off diagonal entries.
} 
with the constant

$$
c_{L}=-\frac{J_{-r-\frac{1}{2}}\left(x_{n L}\right)}{Y_{-r-\frac{1}{2}}\left(x_{n L}\right)} .
$$

Here, $N_{L n}$ is the normalization constant and $x_{n L}=\frac{m_{L n}}{k}$. The functions $J_{\beta}(w)$ and $Y_{\beta}(w)$ appearing in eq.(17) are the Bessel function of the first kind and of the second kind, respectively. On the other hand, the $Z_{2}$ projection condition $Z_{2} \psi=\gamma_{5} \psi$ is used in order to construct the right handed fields on the branes and this ensures that the right handed zero mode appears, the right (left) handed KK modes appear (disappear) on the branes with the boundary conditions:

$$
\begin{aligned}
\left(\frac{d}{d y}+m\right) \chi_{i R n}^{E}\left(y_{0}\right) & =0 \\
\chi_{i L n}^{E}\left(y_{0}\right) & =0 .
\end{aligned}
$$

Again, using the Dirac equation for KK mode leptons, one gets the right handed lepton $\chi_{i R n}^{E}(y)$ that lives on the visible brane as

$$
\chi_{i R n}^{E}(y)=N_{R n} e^{\sigma / 2}\left(J_{\frac{1}{2}+r}\left(e^{\sigma} x_{n R}\right)+c_{R} Y_{\frac{1}{2}+r}\left(e^{\sigma} x_{n R}\right)\right),
$$

with

$$
c_{R}=-\frac{J_{r-\frac{1}{2}}\left(x_{n R}\right)}{Y_{r-\frac{1}{2}}\left(x_{n R}\right)}
$$

where $N_{R n}$ is the normalization constant and $x_{n R}=\frac{m_{R n}}{k}$. Notice that the constant $c_{L}$, the $n^{t h}$ KK mode mass $m_{L n}$ in eq.(17) and the constant $c_{R}$, the $n^{\text {th }} \mathrm{KK}$ mode mass $m_{R n}$ in eq.(20) are obtained by using the boundary conditions eq.(16) and eq.(19), respectively. For $m_{L(R) n} \ll k$ and $k R \gg 1$ they are approximated as:

$$
\begin{array}{ll}
m_{L n} \simeq k \pi\left(n-\frac{\frac{1}{2}-r}{2}+\frac{1}{4}\right) e^{-\pi k R}, & \\
m_{R n} \simeq k \pi\left(n-\frac{\frac{1}{2}+r}{2}+\frac{1}{4}\right) e^{-\pi k R} \quad \text { for } r<0.5, \\
m_{R n} \simeq k \pi\left(n+\frac{\frac{1}{2}+r}{2}-\frac{3}{4}\right) e^{-\pi k R} \quad \text { for } r>0.5 .
\end{array}
$$

Now, we are ready to calculate the $S$-charged lepton-KK charged lepton vertex factors, $V_{L R(R L) i j}^{n}$, that appear in the loop diagram (see Fig,1). The procedure is the integration of the Yukawa interaction eq.(9) over the fifth dimension by taking the $S$-zero mode lepton singlet (doublet)-KK mode lepton doublet (singlet)

$$
V_{R L(L R) i j}^{n}=\frac{\xi_{5 i j}^{E}\left(\left(\xi_{5 i j}^{E}\right)^{\dagger}\right)}{2 \pi R} \int_{-\pi R}^{\pi R} d y \chi_{i R 0(i L 0)}(y) \chi_{j L n(j R n)}(y) \delta(y-\pi R),
$$


and we get

$$
\begin{aligned}
& V_{R L i j}^{n}= \frac{N_{L n} e^{k \pi R\left(1 / 2-r_{i R}\right)}\left(J_{\frac{1}{2}-r_{j L}}\left(e^{k \pi R} x_{n L}\right)+c_{L} Y_{\frac{1}{2}-r_{j L}}\left(e^{k \pi R} x_{n L}\right)\right)}{\pi R \sqrt{\frac{e^{k \pi R\left(1-2 r_{i R}\right)}-1}{k \pi R\left(1-2 r_{i R}\right)}}} \xi_{5 i j}^{E}, \\
& V_{L R i j}^{n}=\frac{N_{R n} e^{k \pi R\left(1 / 2-r_{i L}\right)}\left(J_{\frac{1}{2}+r_{j R}}\left(e^{k \pi R} x_{n R}\right)+c_{R} Y_{\frac{1}{2}+r_{j R}}\left(e^{k \pi R} x_{n R}\right)\right)}{\pi R \sqrt{\frac{e^{k \pi R\left(1-2 r_{i L}\right)}-1}{k \pi R\left(1-2 r_{i L}\right)}}}\left(\xi_{5 i j}^{E}\right)^{\dagger},
\end{aligned}
$$

where $c_{L}\left(c_{R}\right)$ is given in eq.(18) (eq.(21) ) with the replacements $r \rightarrow r_{j L}\left(r \rightarrow r_{j R}\right)$. In the first scenario that the hierarchy of new Yukawa couplings comes from the lepton field location, the effective $S$-zero mode lepton singlet (doublet)-KK mode lepton doublet (singlet) coupling $\xi_{i j}^{E n}\left(\xi_{i j}^{E n}\right)^{\dagger}$ reads

$$
\xi_{i j}^{E n}\left(\left(\xi_{i j}^{E n}\right)^{\dagger}\right)=\frac{V_{R L(L R) i j}^{n}}{V_{R L(L R) \mu \tau}^{0}} \alpha
$$

and, in the second scenario, we get

$$
\xi_{i j}^{E n}\left(\left(\xi_{i j}^{E n}\right)^{\dagger}\right)=\frac{V_{R L(L R) i j}^{n}}{V_{R L(L R) i j}^{0}} \xi_{i j}^{E} .
$$

At this stage, we present the BRs of the $\operatorname{LFV} \mu \rightarrow e \gamma, \tau \rightarrow e \gamma$ and $\tau \rightarrow \mu \gamma$ decays. The existence of decays at least in the one loop level brings the logarithmic divergences in the calculations and we eliminate them by using the on-shell renormalization scheme. In this scheme, the self energy diagrams for on-shell leptons vanish since they can be written as $\sum(p)=\left(\hat{p}-m_{l_{1}}\right) \bar{\sum}(p)\left(\hat{p}-m_{l_{2}}\right)$, and the vertex diagram in Fig.1 gives non-zero contribution.9. Taking only tau lepton for the internal line 10 , the decay width $\Gamma$ reads

$$
\Gamma\left(l_{1} \rightarrow l_{2} \gamma\right)=c_{1}\left(\left|A_{1}\right|^{2}+\left|A_{2}\right|^{2}\right)
$$

where

$A_{1}=Q_{\tau} \frac{1}{48 m_{\tau}^{2}}\left\{6 m_{\tau} \bar{\xi}_{N, \tau l_{2}}^{E} \bar{\xi}_{N, l_{1} \tau}^{E}\left(F\left(z_{h^{0}}\right)-F\left(z_{A^{0}}\right)\right)+\sum_{n=1}^{\infty} m_{l_{1}}\left(\bar{\xi}_{N, l_{2} \tau}^{E} \bar{\xi}_{N, l_{1} \tau}^{E}\left(G\left(z_{h^{0}}\right)+G\left(z_{A^{0}}\right)\right)\right.\right.$

\footnotetext{
${ }^{9}$ This is the case that the divergences can be eliminated by introducing a counter term $V_{\mu}^{C}$ with the relation $V_{\mu}^{\text {Ren }}=V_{\mu}^{0}+V_{\mu}^{C}$, where $V_{\mu}^{R e n}\left(V_{\mu}^{0}\right)$ is the renormalized (bare) vertex and by using the gauge invariance: $k^{\mu} V_{\mu}^{R e n}=0$. Here, $k^{\mu}$ is the four momentum vector of the outgoing photon.

${ }^{10}$ We take into account only the internal tau lepton contribution since, in the second scenario, we respect the idea that the couplings $\bar{\xi}_{N, i j}^{E}(i, j=e, \mu)$, are small compared to $\bar{\xi}_{N, \tau i}^{E}(i=e, \mu, \tau)$, due to the possible proportionality of them to the masses of leptons under consideration in the vertices. In the first scenario, this idea is considered automatically. Here, we use the dimensionful coupling $\bar{\xi}_{N, i j}^{E}$ with the definition $\xi_{N, i j}^{E}=\sqrt{\frac{4 G_{F}}{\sqrt{2}}} \bar{\xi}_{N, i j}^{E}$ where $\mathrm{N}$ denotes the word "neutral".
} 


$$
\begin{aligned}
& \left.\left.+\sum_{n=1}^{\infty} \frac{m_{\tau}^{2}}{m_{n R}^{2}}\left(\bar{\xi}_{N, l_{2} \tau}^{E n}\right)^{\dagger}\left(\bar{\xi}_{N, l_{1} \tau}^{E n}\right)^{\dagger}\left(G\left(z_{n R, h^{0}}\right)+G\left(z_{n R, A^{0}}\right)\right)\right)\right\} \\
A_{2} & =Q_{\tau} \frac{1}{48 m_{\tau}^{2}}\left\{6 m_{\tau} \bar{\xi}_{N, l_{2} \tau}^{E} \bar{\xi}_{N, \tau l_{1}}^{E}\left(F\left(z_{h^{0}}\right)-F\left(z_{A^{0}}\right)\right)+m_{l_{1}}\left(\bar{\xi}_{N, l_{2} \tau}^{E} \bar{\xi}_{N, l_{1} \tau}^{E}\left(G\left(z_{h^{0}}\right)+G\left(z_{A^{0}}\right)\right)\right.\right. \\
& \left.\left.+\sum_{n=1}^{\infty} \frac{m_{\tau}^{2}}{m_{n L}^{2}} \bar{\xi}_{N, l_{2} \tau}^{E n} \bar{\xi}_{N, l_{1} \tau}^{E n}\left(G\left(z_{n L, h^{0}}\right)+G\left(z_{n L, A^{0}}\right)\right)\right)\right\}
\end{aligned}
$$

where $l_{1}\left(l_{2}\right)=\tau ; \mu(\mu$ or $e ; e)$ and the functions $F(w), G(w)$ are given by

$$
\begin{aligned}
& F(w)=\frac{w\left(3-4 w+w^{2}+2 \ln w\right)}{(-1+w)^{3}} \\
& G(w)=\frac{w\left(2+3 w-6 w^{2}+w^{3}+6 w \ln w\right)}{(-1+w)^{4}} .
\end{aligned}
$$

Here $c_{1}=\frac{G_{F}^{2} \alpha_{e m} m_{l_{1}}^{3}}{32 \pi^{4}}, A_{1}\left(A_{2}\right)$ is the left (right) chiral amplitude, $z_{S}=\frac{m_{\tau}^{2}}{m_{S}^{2}}, z_{n L(n R), S}=\frac{m_{n L(n R)}^{2}}{m_{S}^{2}}$ with left (right) handed internal lepton KK mode mass $m_{n L(n R)}$ (eq.(22)), $Q_{\tau}$ is the charge of tau lepton. Notice that we take the Yukawa couplings real and ignore the mass $m_{e}\left(m_{\mu}\right)$ for $\mu \rightarrow e \gamma ; \tau \rightarrow e \gamma(\tau \rightarrow \mu \gamma)$ decays.

\section{Discussion}

The radiative $\mathrm{LFV} l_{i} \rightarrow l_{j} \gamma$ interactions exist at least in the one loop level in the $2 \mathrm{HDM}$, with the help of the Yukawa interactions coming from lepton-lepton- $S$ vertices. The Yukawa couplings are free parameters which should be restricted by using the current and forthcoming experiments. In the present work, we study these decays in the RS1 background with the assumption that the leptons are accessible to the extra dimension. Furthermore, we choose the idea that the lepton fields are localized in the extra dimension by considering a Dirac mass term $m_{l}=r \sigma^{\prime}$ with $\sigma=k|y|$ (eq.(2)). This choice results in that the right and left handed lepton zero modes, the SM leptons, have exponential profiles in the extra dimension, given in eq.(5) , and their different locations can explain different flavor mass hierarchy 11 Here we choose three different set of locations of charged leptons in order to obtain the masses of different flavors. Since we consider that the leptons are accessible to the bulk, the gauge sector of the model should live also in the extra dimension and their KK modes appear after the compactification of the extra dimension. The different fermion locations can induce additional FCNC effects at tree

\footnotetext{
${ }^{11}$ There is another scenario, so called the split fermion scenario, where the mass hierarchy comes from the different locations of various flavors and their left and right handed parts of lepton fields which have Gaussian profiles in the flat extra dimensions. In this case, the localizations of fermions can be obtained by coupling them to a scalar field having kink profile, and the Gaussian profiles of leptons are obtained as an approximation (see [46, 47] for details).
} 
level due to the couplings of neutral gauge KK modes-leptons and they should be suppressed even for low KK masses, by choosing the location parameters $c_{L}\left(c_{R}\right)$ appropriately. In the set of location parameters we use (Table 1), we verify the various experimental FCNC constraints with KK neutral gauge boson masses as low as few TeVs (see the similar the set of location parameters and the discussion given in [43, 44]). On the other hand, in our scenario, since the FV is carried by the new Yukawa couplings which are fixed to an appropriate number, respecting the current measurements, the location parameters of leptons are responsible for the lepton mass hierarcies and the strengths of $S$-zero mode charged lepton-KK mode charged leptonvertices. Therefore, they do not play a crucial role in the existence of the FV in the tree level and the constraints coming from various LFV processes become more relaxed.

In the first set (Table 1), we consider the left and right handed fields having the same location in the extra dimension. This is the case that we construct the hierarchy of new Yukawa couplings by fixing the $\tau-\mu$ coupling $\bar{\xi}_{N, \tau \mu}^{E}$, which is renamed as $\alpha$ in the text. In the second and third set, we choose the left handed charged lepton locations as the same for each flavor and we estimate the right handed ones by respecting the charged lepton masses. In the third set, we take the left handed charged lepton locations near to the visible brane and we observe that the KK mode charged lepton contribution to the BRs of the decays studied increases. The reason behind this enhancement is that the KK mode couplings to the new Higgs scalars, living on the visible brane, become stronger if the left handed lepton field is near to this brane. For the second and third set of locations, we embed the results of integrals of the $S$-zero mode charged lepton-zero mode charged lepton couplings into the Yukawa couplings of corresponding vertices. In this case, we choose the Yukawa couplings such that $\bar{\xi}_{N, i j}^{E}, i, j=e, \mu$ are smaller compared to $\bar{\xi}_{N, \tau i}^{E} i=e, \mu, \tau$, since latter ones contain heavy flavor and we assume that, in four dimensions, the couplings $\bar{\xi}_{N, i j}^{E}$ is symmetric with respect to the indices $i$ and $j$. Furthermore, the curvature parameter $k$ and the compactification radius $R$ are the additional free parameters of the theory. Here we take $k R=10.83$ and consider in the region $10^{17}(\mathrm{GeV}) \leq k \leq 10^{18}(\mathrm{GeV})$ (see the discussion in section 2 and [35]). Throughout our calculations we use the input values given in Table (2).

In Figs 2,34, we present the parameter $k$ dependence of the BR of the LFV decay $\mu \rightarrow$ $e \gamma-\tau \rightarrow e \gamma-\tau \rightarrow \mu \gamma$ for location Set I, for different values of the coupling $\alpha$. In Fig. 2. the solid (dashed, small dashed) line-curve represents the BR for $\alpha=0.1(0.2,0.5) \mathrm{GeV}$ without -with the lepton KK modes. In Figs 3 and 4, the solid (dashed, small dashed) linecurve represents the BR for $\alpha=2(5,10) G e V$ without -with the lepton KK modes, similar to 


\begin{tabular}{|l|l|}
\hline Parameter & \multicolumn{1}{|c|}{ Value } \\
\hline \hline$m_{\mu}$ & $0.106(\mathrm{GeV})$ \\
$m_{\tau}$ & $1.78(\mathrm{GeV})$ \\
$m_{h^{0}}$ & $100(\mathrm{GeV})$ \\
$m_{A^{0}}$ & $200(\mathrm{GeV})$ \\
$G_{F}$ & $1.1663710^{-5}\left(\mathrm{GeV}^{-2}\right)$ \\
\hline
\end{tabular}

Table 2: The values of the input parameters used in the numerical calculations.

the previous figure. Fig 2 shows that the $\mathrm{BR}(\mu \rightarrow e \gamma)$ is at the order of the magnitude of $10^{-13}\left(10^{-12}, 10^{-11}\right)$ for $\alpha=0.1(0.2,0.5) \mathrm{GeV}$ and it is not sensitive to the parameter $k$ in the given range. Here, the enhancement due to the additional contribution coming from internal lepton $\mathrm{KK}$ modes is negligible. The $\mathrm{BR}(\tau \rightarrow e \gamma)-\mathrm{BR}(\tau \rightarrow \mu \gamma)$ is at the order of the magnitude of $10^{-12}\left(10^{-10}, 10^{-9}\right)-10^{-10}\left(10^{-8}, 10^{-7}\right)$ for $\alpha=2(5,10) \mathrm{GeV}$. For these decays the BR slightly increases for the small values of $k, k \sim 10^{17}$ and the enhancement in the $\mathrm{BR}$ due to the lepton KK mode contributions is almost $100 \%$ - more than $100 \%$. To obtain the BR of the decay $\mu \rightarrow e \gamma$ in the range $10^{-14} \leq B R \leq 10^{-11}$, the coupling $\alpha$ should lie in the region $0.1 \leq \alpha \leq 0.5$. On the other hand, this coupling should be in the region $5 \leq \alpha \leq 10$ for the case that the BR of the decay $\tau \rightarrow e \gamma(\tau \rightarrow \mu \gamma)$ with internal lepton KK modes has the numerical value lying in the range $10^{-10} \leq B R \leq 10^{-9}\left(10^{-8} \leq B R \leq 10^{-6}\right)$. These numerical values show that the idea of hierarchy of Yukawa couplings based on the locations of charged leptons in the bulk of RS1 background can not explain the present experimental results of these LFV decays and this stimulates to perform more accurate measurements.

Figs. 5] 6] are devoted to the parameter $k$ dependence of the BR of the LFV $\mu \rightarrow e \gamma$ decay for lepton location set II-III, for $\bar{\xi}_{N, \tau \mu}^{E}=1 G e V$ and for different values of the coupling $\bar{\xi}_{N, \tau e}^{E}$. Here, the solid (dashed, small dashed) line-curve represents the BR for $\bar{\xi}_{N, \tau e}^{E}=0.01(0.005,0.001) \mathrm{GeV}$ without -with the lepton KK modes. It is observed that the $\mathrm{BR}(\mu \rightarrow e \gamma)$ is at the order of the magnitude of $10^{-11}\left(10^{-12}, 10^{-13}\right)$ for $\bar{\xi}_{N, \tau e}^{E}=0.01(0.005,0.001) \mathrm{GeV}$ without the internal lepton KK mode contributions. The addition of lepton KK modes bring considerable enhancement, nearly $3 \times$ one order (nearly two orders) for set II (III), especially for the small values of the parameter $k$. The larger enhancement in the case of set III is due to the fact that the left handed leptons (zero and KK modes) are near to the visible brane and their couplings to the new Higgs scalars, living on the visible brane, become stronger. This is interesting since it would be possible to enhance the BR by localizing the lepton fields at appropriate points in the extra dimension, near to the visible brane. Therefore, by choosing small values of yukawa 
couplings, the experimental values of the BR of the LFV decay under consideration could be reached.

Figs. 7,8 represent the parameter $k$ dependence of the BR of the LFV $\tau \rightarrow e \gamma$ decay for lepton location set II-III, for $\bar{\xi}_{N, \tau e}^{E}=1 G e V$ and for different values of the coupling $\bar{\xi}_{N, \tau \tau}^{E}$. Here, the solid (dashed, small dashed) line-curve represents the BR for and $\bar{\xi}_{N, \tau \tau}^{E}=20(50,80) \mathrm{GeV}$ without -with the lepton KK modes. These figures show that the $\operatorname{BR}(\tau \rightarrow e \gamma)$ is at the order of the magnitude of $10^{-10}\left(10^{-9}, 10^{-8}\right)$ for $\bar{\xi}_{N, \tau \tau}^{E}=20(50,80) \mathrm{GeV}$ without the internal lepton KK mode contributions. With the inclusion of the internal lepton KK modes, there occurs a considerable enhancement, $5 \times$ three order to almost $200 \%$ (more than four orders to $300 \%$ ) in the range of $k, 0.1 \leq k \leq 1$ for set II (III). Even for the large curvature, the enhancement of the BR due to the extra dimension contributions, here, the internal lepton KK modes, is large and this is a worthy observation for checking the existence and, also, the nature of the extra dimensions.

Finally, in Figs. 9-10, we present the parameter $k$ dependence of the BR of the LFV $\tau \rightarrow \mu \gamma$ decay for lepton location set II-III, for $\bar{\xi}_{N, \tau \mu}^{E}=1 G e V$ and for different values of the coupling $\bar{\xi}_{N, \tau \tau}^{E}$. Here, the solid (dashed, small dashed) line-curve represents the BR for and $\bar{\xi}_{N, \tau \tau}^{E}=20(50,80) G e V$ without -with the lepton KK modes. It is observed that the $\mathrm{BR}(\tau \rightarrow \mu \gamma)$ is at the order of the magnitude of $10^{-10}\left(10^{-9}, 10^{-8}\right)$ for $\bar{\xi}_{N, \tau \tau}^{E}=20(50,80) \mathrm{GeV}$ without the internal lepton KK mode contributions. Similar to previous decays, the inclusion of the internal lepton KK modes bring a considerable enhancement to the BR, $5 \times$ three orders to almost $200 \%$ (nearly more than four orders to more than $300 \%$ ) in the range of $k, 0.1 \leq k \leq 1$ for set II (III). The current numerical result $\mathrm{BR}=9.0(6.8) 10^{-8}$ at $90 \% \mathrm{CL}$ by [4] ([5]) can be reached even for weak Yukawa interactions and for intermediate values of the curvature parameter $k$, with the inclusion of lepton KK modes, especially for the location set III.

For completeness, we present the results of the same processes for various scenarios in the case of flat extra dimensions. For the flat one universal extra dimension, the enhancements of the BRs of the LFV decays (especially the BR of the $\mu \rightarrow e \gamma$ decay), due to the lepton KK modes and the new neutral Higgs KK modes are tiny for the compactification radius $R$, $1 / R \geq 500 \mathrm{GeV}$. However, since the contribution of the universal extra dimension to any process exists at least in the loop leve12, the LFV decays, which can exist also in the loop level and which are clean in the sense that are free from the strong interactions, are among the

\footnotetext{
${ }^{12}$ The processes with incoming and outgoing zero mode particles can get contributions from the universal extra dimension at least in the loop level since the KK number should be conserved at the vertices and the tree level contribution with a single KK mode does not appear.
} 
most informative processes in order to search this type of extra dimension. If one considers the flat non-universal extra dimensions where the new Higgs bosons are accessible to the extra dimension but leptons live on the $4 \mathrm{D}$ brane 13 , there exist considerable enhancements in the BRs of the LFV decays, in the case of two spatial extra dimensions and the discrepancy between the experimental and the theoretical values of the BRs can be overcome [11, 12]. In the the split fermion scenario where the fermions are localized with Gaussian profiles at different points in the extra dimension, the enhancements in the BRs of LFV decays are tiny for a single extra dimension and the sensitivities of the BRs increase for two extra dimensions [13]. For both cases, the enhancements in the BRs are due to the abundance of new neutral Higgs KK modes. If the new Higgs doublet is restricted to the 4D brane (thin bulk), we observe that the BRs are sensitive to the location of the $4 \mathrm{D}$ brane in the extra dimension (the width of the thin bulk), especially for the $\mu \rightarrow e \gamma$ decay [14]. Notice that in the case of non-universal extra dimensions, there is no need for the KK number to conserve at the vertices and the processes with a single internal KK mode can exist. Therefore, the non-universal extra dimension(s) results in non zero contribution also in the tree level and the physical quantities related to such processes are not suppressed. Even if the BRs of the LFV interactions are sensitive to the effects of the non-universal extra dimensions, the processes existing in the tree level with internal KK modes are more informative to search the extra dimensions.

Now we would like to present our results briefly.

- The idea that the hierarchy of new Yukawa couplings is described by the location of leptons in the bulk of RS1 background is worthwhile to study. However, with a unique set of Yukawa couplings, the coupling $\alpha$ and the others created from $\alpha$, we observe that it is not possible to get theoretical values of the BRs coinciding with the current experimental results of the LFV decays under consideration. Furthermore, we see that the BRs are not sensitive to the curvature parameter $k$ and the internal lepton KK mode contributions. Notice that, in this case, we choose the left handed and right handed charged lepton positions as the same in the extra dimension. The future more accurate experimental measurements, especially for the $\tau \rightarrow e \gamma$ decay, would make it possible to test the physics behind the hierarchy of new Yukawa couplings and the effect of extra dimensions on this hierarchy.

- In the case that the left handed and right handed charged lepton positions are different in

\footnotetext{
${ }^{13}$ The electric dipole moments of the charged leptons are among the clean physical quantities and they are worthwhile to study in order to search the extra dimensions.
} 
the extra dimension, the addition of lepton KK modes result in considerable enhancement in the BRs, especially for the small values of the parameter $k$. Furthermore, this enhancement increases and the sensitivity of $\mathrm{BR}$ to the curvature parameter $k$ becomes strong by taking the location points of left handed charged leptons near to the visible brane. This is due to the fact that the left handed KK mode couplings to the new Higgs scalars, living on the visible brane, become stronger. Therefore, it would be possible to enhance the BRs by localizing the lepton fields at appropriate points in the extra dimension, and, even with the small values of Yukawa couplings and even in single extra dimension, the experimental values of the BR of the LFV decay under consideration can be reached. This is interesting and the forthcoming measurements of the BRs of the LFV decays, especially the $\mu \rightarrow e \gamma$ decay, would give considerable information about the existence and, also, the nature of the warped extra dimensions. .

\section{References}

[1] M. L. Brooks et. al., MEGA Collaboration, Phys. Rev. Lett. 83 1521, (1999).

[2] K. Hayasaka et al.., Phys.Lett. D63 20, (2005).

[3] S. Ahmed et.al., CLEO Collaboration, Phys. Rev. D61 071101, (2000).

[4] J.M. Roney and the BABAR Collaboration, Nucl. Phys. Proc. Suppl. 144 155, (2005).

[5] B. Aubert et. al., BABAR Collaboration, SLAC-PUB-11028, BABAR-PUB-04-049, Feb. 2005, 7. pp, Phys. Rev. Lett. 95 041802, (2005).

[6] Donato Nicolo, MUEGAMMA Collaboration, Nucl. Instrum. Meth A503 287, (2003).

[7] S. Yamada, Nucl. Phys. Proc. Suppl. 144 185, (2005).

[8] E. O. Iltan, Phys. Rev. D64 115005, (2001).

[9] E. O. Iltan,Phys. Rev. D64 013013, (2001)

[10] R. Diaz, R. Martinez and J-Alexis Rodriguez, Phys.Rev. D63 095007, (2001).

[11] E. O. Iltan, JHEP 0402 20, (2004).

[12] E. O. Iltan, JHEP 0408 20, (2004) 
[13] E. O. Iltan, Mod. Phys. Lett. A22 819, (2007).

[14] E. O. Iltan, Eur. Phys. J. C46 475, (2006).

[15] R. Barbieri and L. J. Hall, Phys. Lett. B338 212, (1994).

[16] R. Barbieri, L. J. Hall and A. Strumia, Nucl. Phys. B445 219, (1995).

[17] R. Barbieri, L. J. Hall and A. Strumia, Nucl. Phys. B449 437, (1995).

[18] P. Ciafaloni, A. Romanino and A. Strumia, IFUP-YH-42-95.

[19] T. V. Duong, B. Dutta and E. Keith, Phys. Lett. B378 128, (1996).

[20] G. Couture, et. al., Eur. Phys. J. C7 135, (1999).

[21] Y. Okada, K. Okumara and Y. Shimizu, Phys. Rev. D61 094001, (2000).

[22] D. Chang, W. S. Hou and W. Y. Keung, Phys. Rev. D48 217, (1993).

[23] P. Paradisi, JHEP 0602 050, (2006).

[24] L. Randall, R.Sundrum, Phys. Rev. Lett. 83 3370, (1999).

[25] L. Randall, R.Sundrum, Phys. Rev. Lett. 834690 (1999);

[26] W. D. Goldberger, M. B. Wise, Phys. Rev. Lett. D 83 4922, (1999).

[27] S. Chang, J. Hisano, H. Nakano, N. Okada, M. Yamaguchi, Phys. Rev. D62 084025, (2000).

[28] A. Pomarol, Phys. Lett. B486 153, (2000).

[29] H. Davoudias, J. L. Hewett, T. G. Rizzo, Phys. Lett. B473 43, (2000).

[30] T. Gherghetta, A. Pomarol, Nucl. Phys. B586 141, (2000).

[31] B. Batell, T. Gherghetta, Phys. Rev. D73, 045016 (2006).

[32] S. J. Huber,C. A. Lee, Q. Shafi, Phys. Lett. B531 112, (2002).

[33] Y. Grossman, M. Neubert, Phys. Lett. B474 361, (2000).

[34] S. J. Huber, hep-ph/0211056, (2002).

[35] S. J. Huber, Nucl. Phys. B666 269, (2003). 
[36] S. J. Huber, Q. Shafi, Phys. Rev. D63 045010, (2001).

[37] I. I. Kogan, S. Mouslopolous, A. Papazoglou, G. G. Ross, Nucl. Phys. B615 191, (2001).

[38] T. Gherghetta, A. Pomarol, Nucl. Phys. B602 3, (2001).

[39] K. Ghoroku, A. Nakamura, Phys. Rev. D65 084017, (2002).

[40] K. Agashe, G. Perez and A. Soni, Phys. Rev. D71 016002, (2005).

[41] K.Agashe, A. E. Blechman and F. Petriello, Phys. Rev. D74 053011, (2006).

[42] E. D. Pree, M. Sher., Phys. Rev. D73, 0955006 (2006).

[43] G. Moreau, J. I. S. Marcos, JHEP. 0603, 090 (2006)

[44] F. Ledroit, G. Moreau, J. Morel, hep-ph/0703262 (2007).

[45] T. P. Cheng and M. Sher, Phy. Rev. D35 3383, (1987).

[46] N. Arkani-Hamed and M. Schmaltz, Phys. Rev. D61 033005, (2000).

[47] E. A. Mirabelli, Schmaltz, Phys. Rev. D61 113011, (2000). 


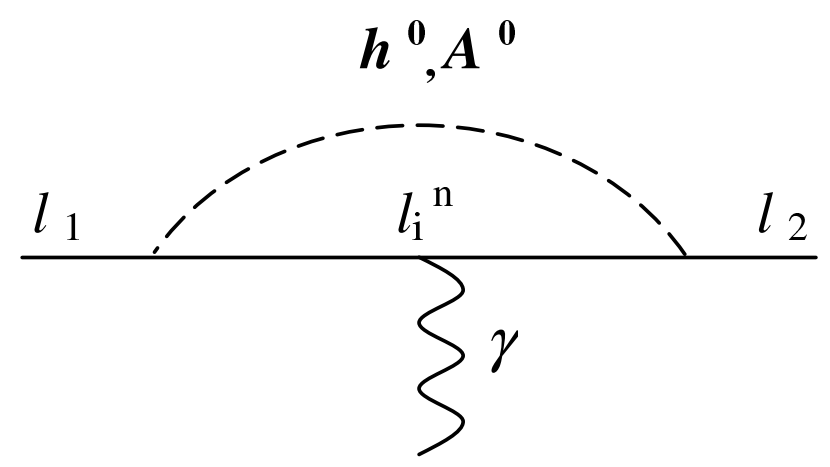

Figure 1: One loop diagrams contribute to $l_{1} \rightarrow l_{2} \gamma$ decay due to the zero mode (KK mode) leptons in the $2 \mathrm{HDM}$. 


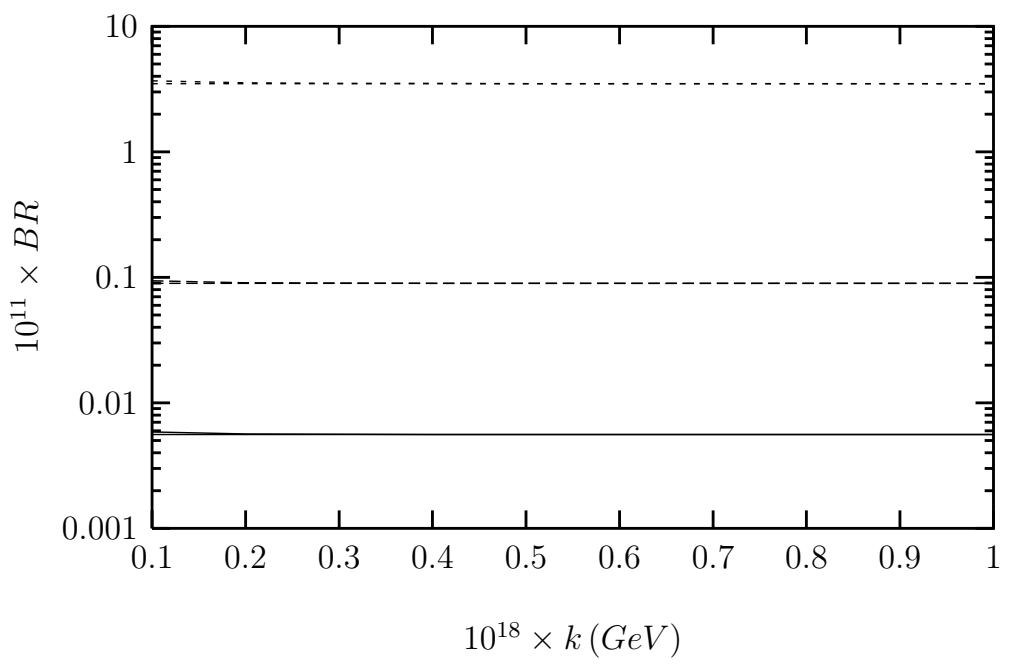

Figure 2: The parameter $k$ dependence of the BR of the LFV $\mu \rightarrow e \gamma$ decay for the location Set I and different values of the coupling $\alpha$. The solid (dashed, small dashed) line-curve represents the BR for $\alpha=0.1(0.2,0.5) \mathrm{GeV}$ without-with the lepton KK modes.

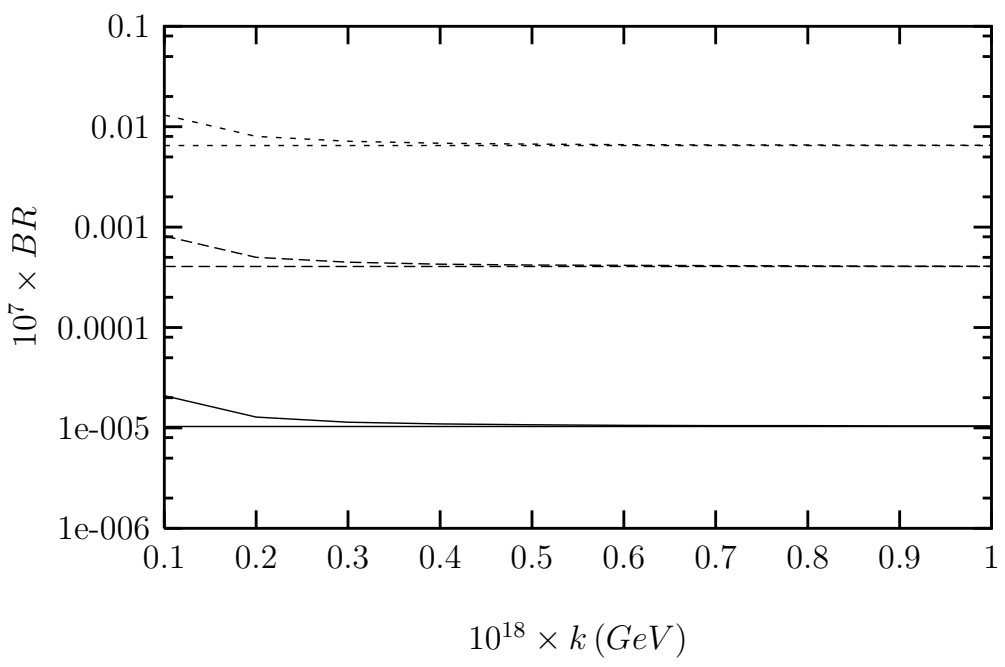

Figure 3: The same as Fig, 2 but for $\tau \rightarrow e \gamma$ decay and $\alpha=2(5,10) \mathrm{GeV}$. 


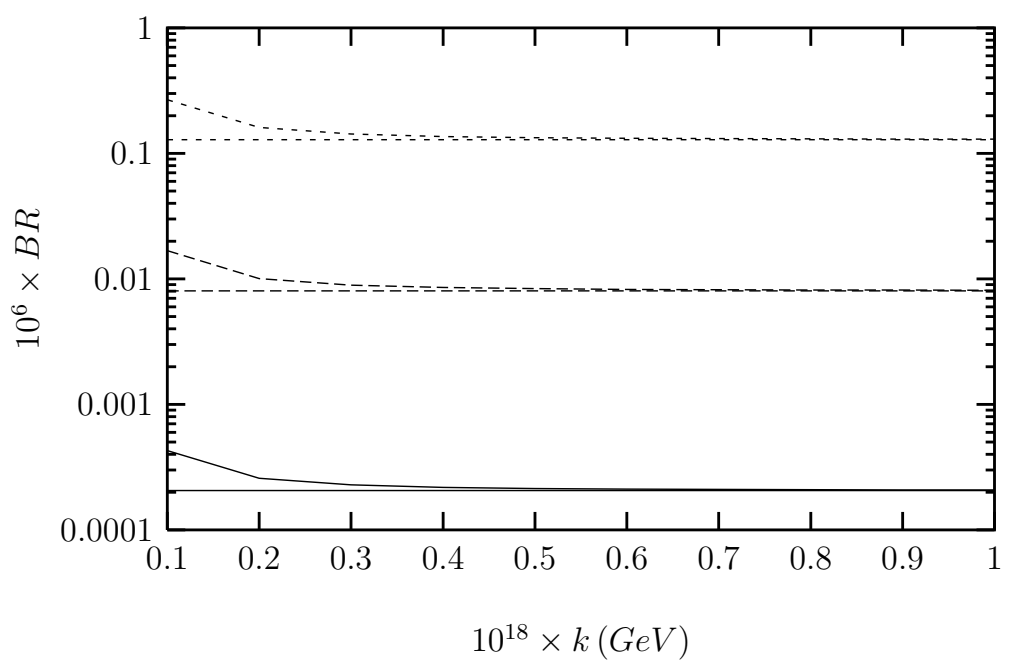

Figure 4: The same as Fig 3 but for $\tau \rightarrow \mu \gamma$ decay.

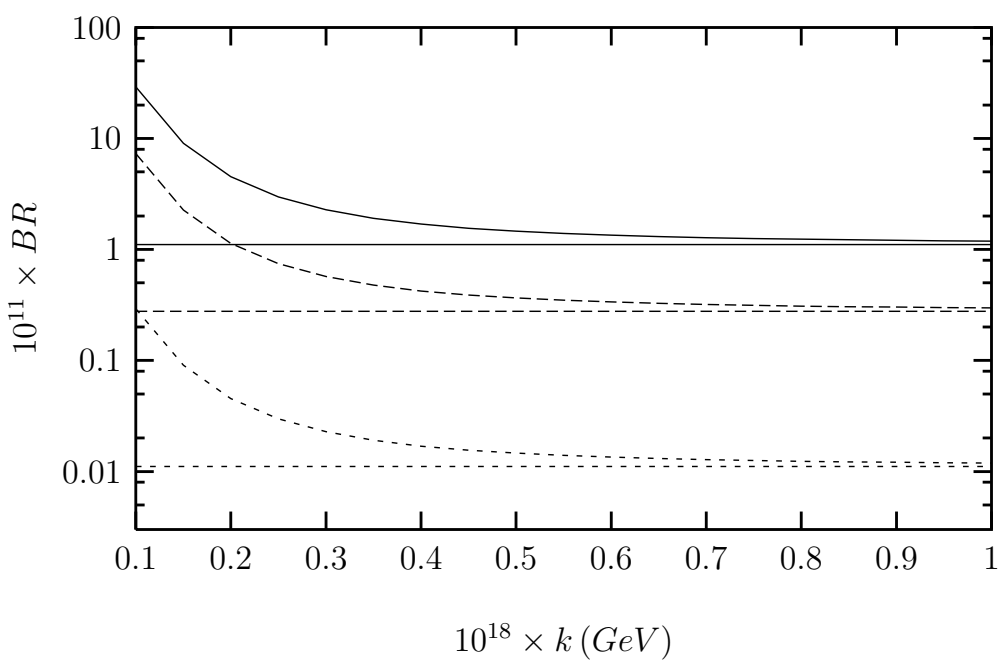

Figure 5: The parameter $k$ dependence of the BR of the LFV $\mu \rightarrow e \gamma$ decay for the location Set II, for $\bar{\xi}_{N, \tau \mu}^{E}=1 \mathrm{GeV}$ and for different values of the coupling $\bar{\xi}_{N, \tau e}^{E}$. Here, the solid (dashed, small dashed) line-curve represents the BR for and $\bar{\xi}_{N, \tau e}^{E}=0.01(0.005,0.001) \mathrm{GeV}$ without -with the lepton KK modes. 


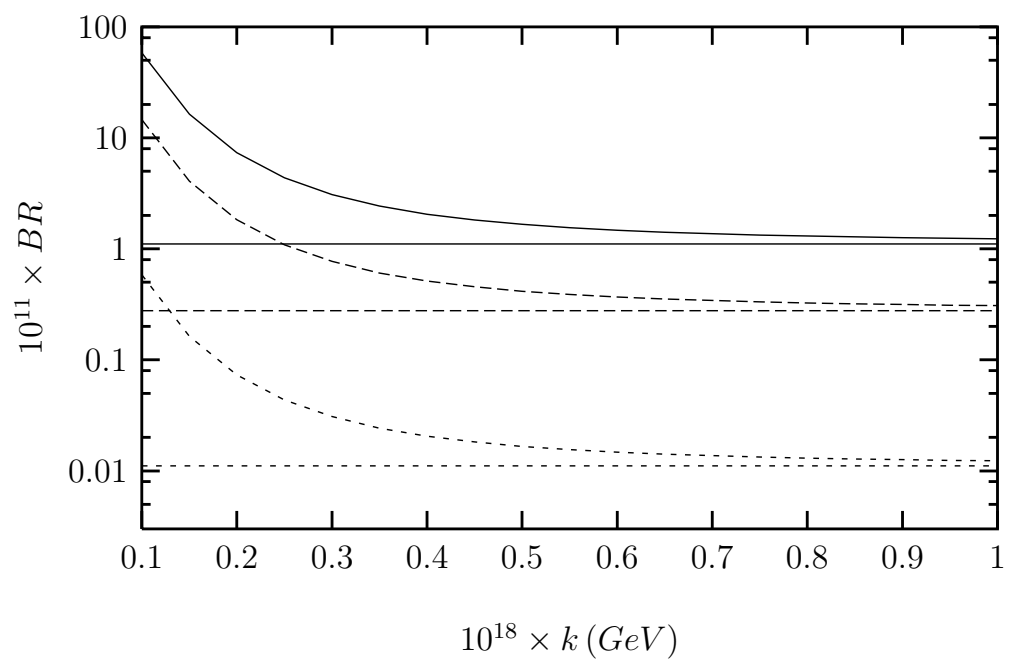

Figure 6: The same as Fig. 5 but for the location Set III.

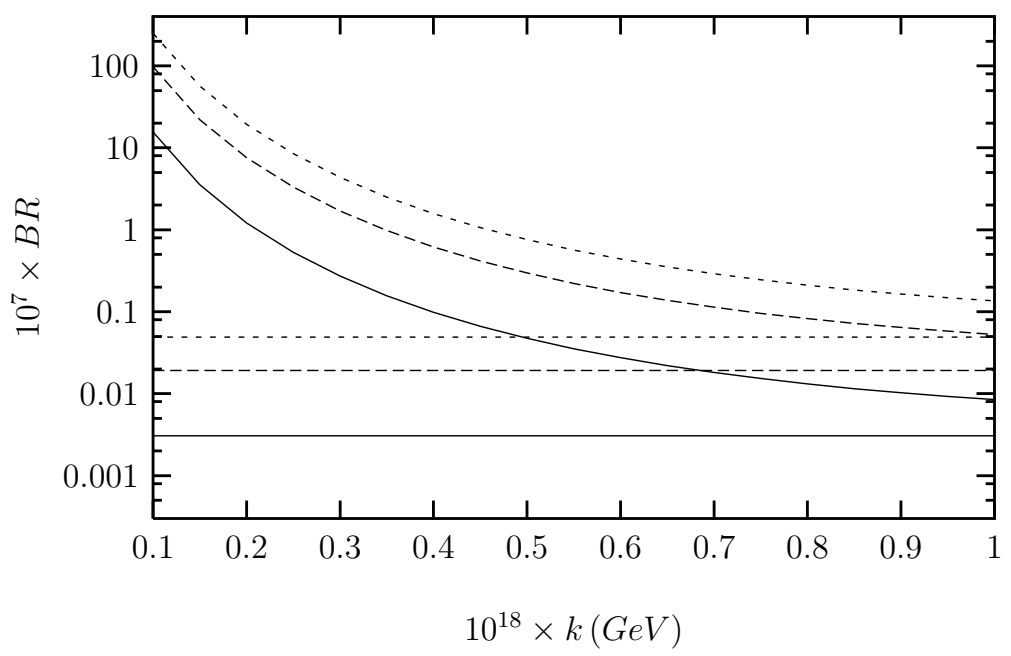

Figure 7: The parameter $k$ dependence of the BR of the LFV $\tau \rightarrow e \gamma$ decay for the location Set II, for $\bar{\xi}_{N, \tau e}^{E}=1 \mathrm{GeV}$ and for different values of the coupling $\bar{\xi}_{N, \tau \tau}^{E}$. Here, the solid (dashed, small dashed) line-curve represents the BR for and $\bar{\xi}_{N, \tau \tau}^{E}=20(50,80) \mathrm{GeV}$ without-with the lepton KK modes. 


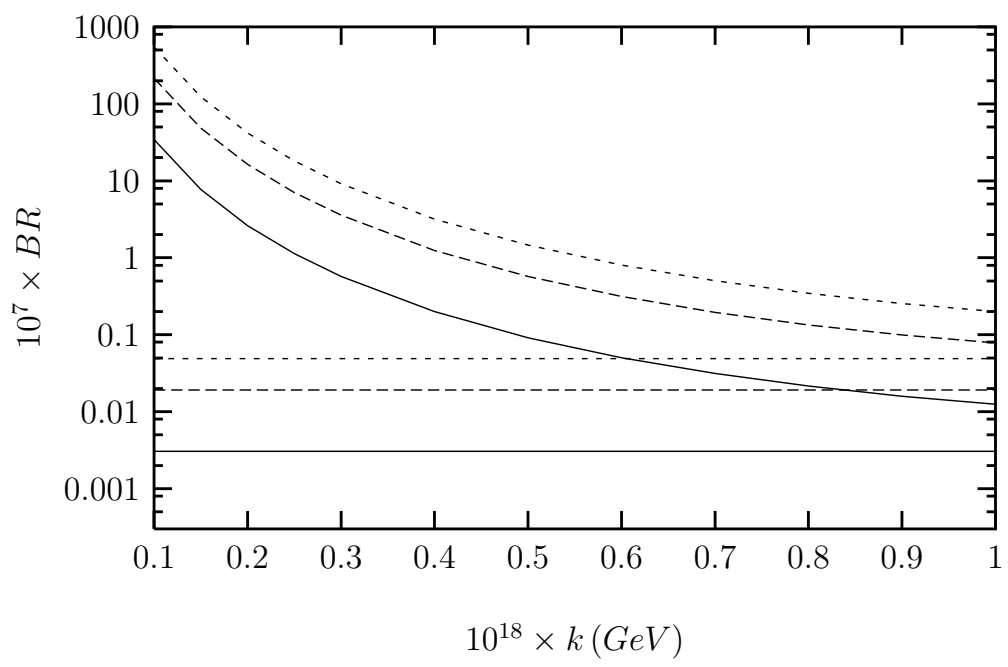

Figure 8: The same as Fig:7 but for the location Set III.

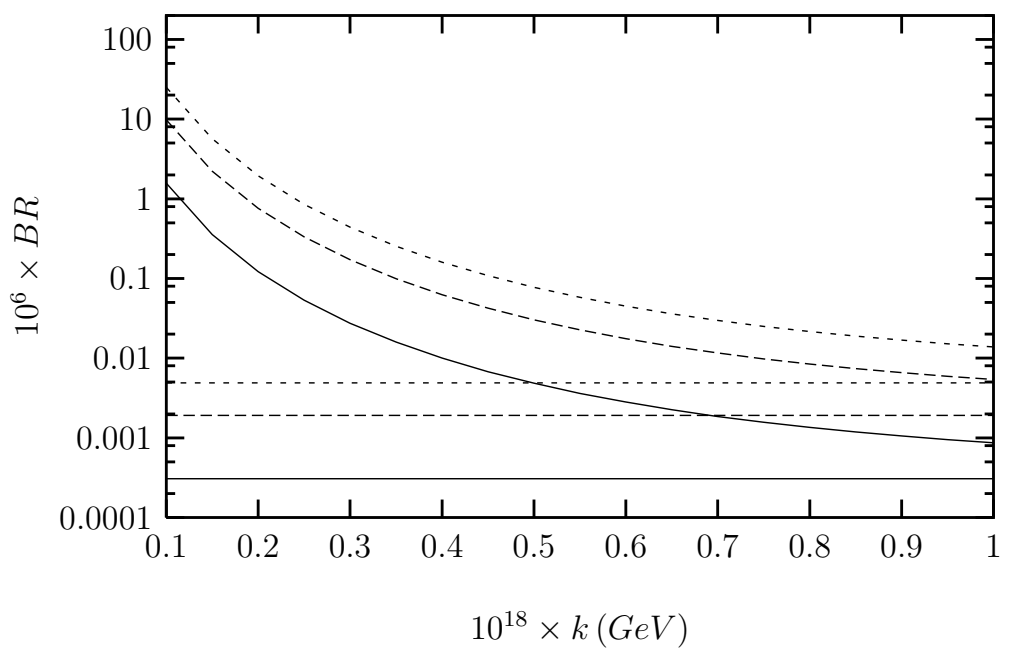

Figure 9: The parameter $k$ dependence of the BR of the LFV $\tau \rightarrow \mu \gamma$ decay for the location Set II, for $\bar{\xi}_{N, \tau \mu}^{E}=1 \mathrm{GeV}$ and for different values of the coupling $\bar{\xi}_{N, \tau \tau}^{E}$. Here, the solid (dashed, small dashed) line-curve represents the BR for and $\bar{\xi}_{N, \tau \tau}^{E}=20(50,80) \mathrm{GeV}$ without -with the lepton KK modes. 


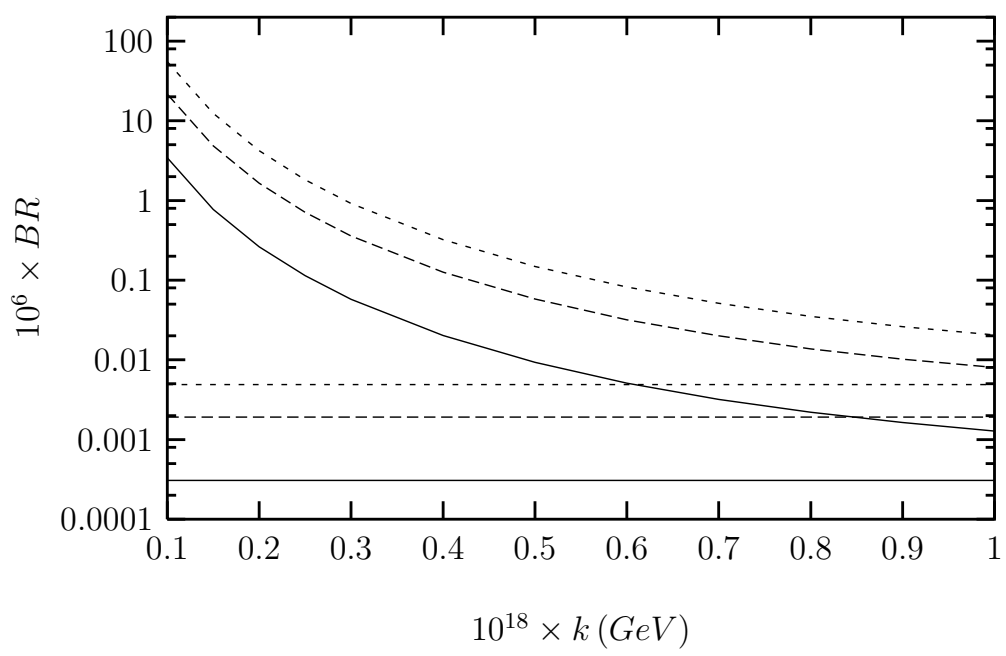

Figure 10: The same as Fig.9 but for the location Set III. 